\title{
DIVERSIDAD DE RAZAS DE Colletotrichum lindemuthianum EN ANTIOQUÍA Y EVALUACIÓN DE GERMOPLASMA DE FRIJOL CREMA-ROJO POR RESISTENCIA A ANTRACNOSIS1
}

\author{
Gloria E. Santana ${ }^{2}$, George Mahuku
}

\begin{abstract}
RESUMEN
Diversidad de razas de Colletotrichum lindemuthianum en Antioquía y evaluación de germoplasma de frijol crema-rojo por resistencia a antracnosis. Para verificar si la composición de razas en el Oriente de Antioquía se caracterizaron 15 aislamientos de $C$. lindemuthianum, usando 12 variedades diferenciales de frijol aceptadas internacionalmente. Se identificaron 11 razas, seis de las cuales $(9,131,135,139$, 643, y 645) fueron caracterizadas por primera vez. Además, se evaluaron 49 genotipos procedentes de los bancos de germoplasma del CIAT y CORPOICA y cultivares de agricultor de municipios productores. Los genotipos fueron evaluados en campo y en invernadero con las razas más virulentas del patógeno (651 y 653) encontradas en Antioquía. Sólo cuatro genotipos (AND1084, SUG130, LAS106 y DiacolCatio) mostraron resistencia a ambas razas. Estos genotipos podrían usarse como parentales para un plan de cruzas, con el fin de introducir genes de resistencia a variedades de importancia comercial que presenten susceptibilidad a la antracnosis.
\end{abstract}

\begin{abstract}
Diversity of Colletotrichum lindemuthianum races in Antioquia and evaluation of cream-red bean germplasm for anthracnose resistance. To verify the composition of Colletotrichum lindemuthianum races in east of Antioquia, 12 differential bean varieties were used to characterize 15 isolates collected from naturally infected bean varieties. Eleven races were identified among the 15 isolates characterized, six of which (races 9, 131, 135, 139, 643, y 645) were characterized for the first time. In addition, we evaluated 49 bean genotypes collected from the bean genebank of CIAT and CORPOICA, as well as landraces from farmers in producing. These genotypes were evaluated under natural field conditions as well as in the greenhouse, using races 651 and 653, previously identified as the most virulent races from Antioquia. Only four genotypes (AND1084, SUG130, LAS106 and Diacol Catio) were resistant under both field and greenhouse conditions. These genotypes can be used as parents in breeding programs to introduce anthracnose resistant genes to bean genotypes with commercial seed types.
\end{abstract}

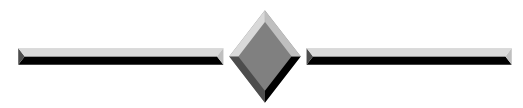

\section{INTRODUCCIÓN}

El frijol común (Phaseolus vulgaris L.) es la leguminosa de grano más importante en América Latina (Pastor y Tu 1989) y en Colombia bajo un ámbito amplio de sistemas de cultivo y ambientes que favorecen los tipos volubles llegando a ser cerca del $65 \%$ de la producción nacional. Antioquía es uno de los primeros departamentos productores de frijol en Colombia con los tipos radical y Cargamanto. Este último es de gran importancia por el tipo voluble, por ser cultivado por más de 30.000 familias en cerca de 26.000 ha/año. Además, este genotipo único en el mundo pertenece a la raza Perú que se caracteriza por tener una semilla grande (50-80 g/100 semillas) y redonda, un ciclo largo de vida (130-140 días) y un alto rendimiento en

\footnotetext{
1 Recibido para publicación el 11 de junio del 2000. Presentado en la XLVI Reunión Anual del PCCMCA, San Juan, Puerto Rico, 2000.

2 Centro de Investigación "La Selva". CORPOICA (Corporación Colombiana de Investigación Agropecuaria). A.A. 100 Rionegro, Antioquia, Colombia. E-mail:hopesantana@yahoo.es; corpoic@epm.net.co

3 CIAT (Centro Internacional de Agricultura Tropical). Apdo. 6713 Cali, Valle, Colombia. E-mail: g.mahuku@cgiar.org
} 
ambientes favorables (Singh 1989, Voysest 1999); así mismo, es una fuente de proteína, fibras naturales, minerales y vitaminas y forma parte del sustento y la dieta diaria de la población rural colombiana. A pesar de todas estas bondades, los genotipos regionales de gran aceptación comercial son altamente susceptibles a enfermedades endémicas como la antracnosis.

La antracnosis, causada por el hongo Colletotrichum lindemuthianum (Sacc \& Mag.) Lams. - Scrib, es probablemente la enfermedad más importante de Phaseolus vulgaris y puede llegar a causar pérdidas en rendimiento de hasta un $95 \%$ cuando se siembran variedades susceptibles. Esta enfermedad afecta principalmente la calidad de la vaina de las plantas de frijol, forma lesiones carnosas, coloreadas, que luego se transforman en chancros hundidos (Chaves 1989). Así mismo, las semillas infectadas frecuentemente presentan decoloración y pueden formar chancros cafés a negros (Chaves 1980). Su severidad induce a muchos agricultores a la utilización de una amplia variedad de fungicidas, lo cual representa altos costos en la producción y contaminación ambiental (Román 1992).

Una de las estrategias más prácticas y deseables para manejar la antracnosis es el uso de variedades de frijol resistentes a la enfermedad. Sin embargo, es conocido que $C$. lindemuthianum es altamente variable (Pastor-Corrales 1992, Tamayo et al. 1995). Los estudios de la diversidad de antracnosis presentes en Antioquía han demostrado la presencia de 11 razas, entre las cuales la 651 y 653 son las más virulentas (Restrepo 1994, Tamayo et al. 1995). No se conoce si en condiciones de campo los cambios que han ocurrido en la estructura de $C$. lindemuthianum, o la aparición de nuevas razas por mutación o por inmigración, pueden afectar la reacción de las variedades de frijol a este patógeno. El conocimiento de la variabilidad genética del patógeno facilita la identificación de genotipos resistentes que podrían ser usados en esta región.

Debido a que el $90 \%$ del frijol Cargamanto cultivado para la producción comercial pertenece a variedades criollas susceptibles a la antracnosis y dado que Colletotrichum lindemuthianum sobrevive en los residuos de cosecha en el suelo y en la semilla por muchos años, el programa de mejoramiento genético de frijol voluble tipo Cargamanto para las zonas de producción de clima frío moderado desarrollado por CORPOICA junto con el apoyo científico del CIAT, ha establecido como prioridad desarrollar cultivares de tipo comercial con resistencia a esta enfermedad y altos rendimientos. Los objetivos de esta investigación fueron: (1) Evaluar germoplasma de frijol común (Phaseolus vulgaris L.) tipo crema-rojo moteado, procedente de los bancos de germoplasma de frijol de CORPOICA (Corporación
Colombiana de Investigación Agropecuaria, Antioquía, Colombia) y CIAT (Centro Internacional de Agricultura Tropical, Cali, Colombia) y de los cultivos comerciales de las zonas productoras tradicionales con el fin de analizar la resistencia a la antracnosis y (2) Verificar los cambios en la población de $C$. lindemuthianum en el departamento de Antioquía, y utilizar esta información en el desarrollo de variedades de frijol con resistencia duradera a la antracnosis.

Los resultados aquí presentados demuestran claramente la diversidad de $C$. lindemuthianum, lo cual evidencia la evolución del patógeno, y las potenciales fuentes de resistencia a esta enfermedad que podrían ser usadas en los programas de mejoramiento para este tipo de frijol.

\section{MATERIALES Y MÉTODOS}

\section{Material vegetal}

Con base en genotipos de frijol previamente identificados por su alta resistencia a las cepas de $C$. lindemuthianum bajo condiciones de campo e invernadero en Popayán, Colombia, se seleccionaron 26 del tipo crema-rojo moteado, del banco de germoplasma de frijol del CIAT (Centro Internacional de Agricultura Tropical, Cali, Colombia), de los cuales 16 genotipos fueron volubles y 10 arbustivos. Así mismo, se seleccionaron nueve genotipos del tipo crema-rojo moteado del banco de germoplasma de CORPOICA (Corporación Colombiana de Investigación Agropecuaria, Antioquía, Colombia); de los cuales tres variedades fueron volubles tipo Cargamanto mejoradas, (ICA Llanogrande, frijol ICA L.S -3.3, e ICA Viboral) y dos variedades arbustivas (DiacolCatío variedad mejorada y Mocho La Ceja variedad Regional). Igualmente se colectaron nueve cultivares de agricultor o comerciales tipo Cargamanto en los municipios de Guarne, El Carmen de Viboral, Marinilla, San Vicente y Rionegro, productores de frijol por tradición.

\section{Aislamiento de los hongos}

Los aislamientos de $C$. lindemuthianum caracterizados en este estudio fueron obtenidos de vainas y hojas de genotipos de frijol naturalmente infectados en el Centro de Investigación La Selva en Rionegro, que mostraban síntomas típicos de la antracnosis y enviados para su procesamiento al CIAT; los genotipos comerciales conservaron la identificación del municipio y el nombre de la variedad o genotipo del sitio de procedencia original. El aislamiento del patógeno y la producción 
de cultivos monospóricos se realizó de acuerdo al protocolo usado por Otoya et al. (1995). Los tejidos infectados (vainas y hojas) fueron colocados en una cámara húmeda con el fin de favorecer la esporulación del patógeno. Posteriormente una muestra de conidias de las lesiones esporuladas fueron mezcladas con agua destilada estéril y esparcidas en medio Papa Dextrosa Agar (PDA). Este se incubó a $20^{\circ} \mathrm{C}$ durante 24 horas, al cabo de las cuales las esporas germinadas y bien separadas fueron transferidas a otro medio PDA para continuar el desarrollo del cultivo monospórico.

\section{Producción del inóculo y caracterización de razas}

El inóculo fue producido a partir de los cultivos monospóricos de $C$. lindemuthianum crecidos en medio PDA. El hongo fue incubado a $20^{\circ} \mathrm{C}$ durante siete días, al cabo de los cuales las esporas fueron suspendidas en $5 \mathrm{ml}$ de agua destilada estéril. Esto se hizo raspando delicadamente la superficie del hongo mediante un asa estéril con el fin de obtener la mayor cantidad posible de esporas y filtrando la solución obtenida a través de una gasa estéril para así remover los fragmentos de micelio. Un hemacitómetro fue usado para medir la concentración de esporas, la cual se ajustó a una concentración final de $1,2 \times 10^{6}$ conidias $/ \mathrm{ml}$. Cada cultivo monospórico fue caracterizado de acuerdo a su patotipo de virulencia usando un juego de 12 variedades diferenciales de frijol (Pastor 1992). Esta inoculación se realizó en plantas de frijol con aproximadamente ocho días después de la emergencia, al asperjarlas con la suspensión de conidias e incubarlas en una cámara húmeda a $22^{\circ} \mathrm{C}$ y $90-100 \%$ de humedad relativa. Ocho días después de la infección las plantas fueron evaluadas usando la escala CIAT (Schoonhoven y Pastor 1987). La inoculación se repitió para cada aislamiento. La reacción de las variedades diferenciales se clasificó como reacción incompartible o resistencia si la planta no mostraba síntomas visibles de la enfermedad o si sólo tenía pequeñas lesiones sin esporulación, o reacción compatible o de susceptibilidad si la planta mostraba lesiones típicas de antracnosis. Si el promedio de todas las evaluaciones estuvo entre uno y tres el genotipo fue calificado como resistente (R). Los genotipos que tuvieron una reacción intermedia entre cuatro y seis fueron clasificados como susceptibles (S); si la evaluación de los síntomas fue mayor a seis el genotipo fue calificado como susceptible (S). Para designar o nombrar la raza, a cada variedad diferencial se le dio un valor numérico basado en el sistema binario. La designación de la raza se obtuvo de la sumatoria de los valores binarios de las variedades diferenciales susceptibles.

Las variedades diferenciales con su valor binario (en parentesis) se presentan a continuación: A = Miche- lite (1); $\mathrm{B}=$ MDRK (2); $\mathrm{C}=$ Perry Marrow (4); $\mathrm{D}=$ Cornell 49242 (8); E = Widusa (16); F = Kaboon (32); $\mathrm{G}=$ México 222 (64); H = PI 207262 (128); $\mathrm{I}=\mathrm{TO}$ (256); J = TU (512); K = AB 136 (1024); L = G2333 (2048).

\section{Evaluación de genotipos en campo}

Los 49 genotipos fueron sembrados durante el segundo semestre de 1998 y el primer semestre de 1999 en el Centro de Investigación "La Selva", localizado a $2.100 \mathrm{msnm}$, con una temperatura promedio de $17^{\circ} \mathrm{C}$ y una precipitación anual de $1.700 \mathrm{~mm}$. Se utilizó un diseño experimental de bloques completos al azar con dos repeticiones; en cada bloque se sembraron 11 semillas de cada genotipo con una distancia entre plantas de 0,25 $\mathrm{m}$; en surcos de tres metros de longitud y 0,70 metros de ancho. Entre los surcos de los genotipos a evaluar se sembró un surco testigo, este último para los materiales volubles fue la variedad ICA Viboral y para los arbustivos la variedad regional Mocho La Ceja.

Durante el período de cultivo los genotipos se manejaron de acuerdo con las prácticas culturales establecidas para el monocultivo de frijol en Antioquía; el manejo fitosanitario del cultivo se realizó mediante el uso de fungicidas para el control de la mancha ascochyta o anillada producida por el hongo Phoma exigua var. Diversispora, mientras que no se realizó ningún control químico para la antracnosis.

La evaluación para la antracnosis se realizó utilizando la escala CIAT (Schoonhoven y Pastor 1987, Tamayo 1995), la cual utiliza un rango de calificación de $1=\sin$ síntomas aparentes de la enfermedad hasta $9=$ síntomas severos o muerte, en las etapas de desarrollo V2, V4, R6, R7 y R8. Se consideraron genotipos resistentes (R) a la antracnosis, aquellos que presentaron promedios entre uno y tres en la evaluación de síntomas; genotipos con una reacción intermedia (I) cuando la evaluación de los síntomas estuvo entre cuatro y seis; y los genotipos con promedios entre siete y nueve fueron considerados susceptibles (S).

\section{Evaluación de genotipos en invernadero}

Las evaluaciones de los genotipos bajo condiciones de invernadero se realizaron con las razas más virulentas de $C$. lindemuthianum (los diferenciales ABDHJ mostraron susceptibilidad a la raza 651 mientras que la raza 653 atacó a los genotipos acdhj) para Antioquía de acuerdo a la colecta e identificación previa de razas de antracnosis provenientes de esta zona productora y guardadas en la unidad de patología de 
frijol del CIAT. Se sembraron las semillas de cada genotipo en el Centro Internacional de Agricultura Tropical (CIAT, Cali, Colombia), utilizando un diseño completamente al azar. Siete días después de la siembra, las hojas primarias de cada una de las plantas fueron inoculada por el haz y el envés con una suspensión de esporas de $C$. lindemuthianum (1,2 X $10^{6}$ conidias $\left./ \mathrm{ml}\right)$. La suspensión de esporas de las razas 651 y 653 fueron aplicadas de forma independiente a 10 plantas por genotipo con la ayuda de un compresor. Posteriormente las plantas fueron incubadas por siete días en una cámara húmeda, la cual fue mantenida a una temperatura promedio de 20 a $22^{\circ} \mathrm{C}$ y al $100 \%$ de humedad relativa. Cada evaluación tuvo dos repeticiones. La reacción del genotipo de acuerdo a cada raza inoculada, se obtuvo al promediar la evaluación visual de los síntomas usando la escala CIAT (Schoonhoven y Pastor 1987).

\section{RESULTADOS Y DISCUSIÓN}

\section{Diversidad de razas en Antioquía}

La caracterización de virulencia de los 15 aislamientos provenientes de plantas colectadas en condiciones naturales de campo, mostraron 11 razas (Cuadro 1). Cinco de las cuales $(1,5,129,513$, y 641) habían sido registradas antes en el mismo departamento (PastorCorrales et al., 1993; Otoya et al., 1995; Restrepo,
1994; Tamayo et al., 1995). La raza nueve se caracterizó por primera vez en esta región aunque ya había sido caracterizada en otros departamentos de Colombia (Valle de Cauca, Cundinamarca, Nariño, y Cauca) (Restrepo 1994). Las cinco razas restantes $(131,135,139,643$ y 645) se identificaron por primer vez en Colombia. Dieciséis razas fueron caracterizadas previamente en el departamento de Antioquía (Rionegro), de cuales las razas 651 y 653 se mostraron como las más virulentas (Restrepo 1994, Tamayo et al. 1995). Ninguna de estas dos razas fueron identificadas en este estudio. El ámbito de razas que existe en Antioquía demuestra la compleja estructura de las razas de $C$. lindemuthianum. Además, este resultado muestra que la estructura de razas de $C$. lindemuthianum en el Oriente de Antioquía está cambiando. Un estudio continuo es necesario para estudiar la estructura del patógeno. El alto número de razas identificadas muestra que $C$. lindemuthianum es altamente variable en la región.

\section{Reacción de genotipos procedentes de campos co- merciales}

Los resultados de las evaluaciones en campo confirman que todos los genotipos comerciales utilizados por los productores son susceptibles a la antracnosis al igual que el testigo ICA Viboral (Cuadro 2). Quirós et al. (1996) y Arias y Monsalve (2000) reportan la frecuente selección de la semilla después de la cosecha,

Cuadro 1. Evaluación de la severidad a la antracnosis de nueve genotipos de frijol tipo Cargamanto colectados en cultivos comerciales, en condiciones de campo e invernadero. Antioquía, Colombia. 1999.

\begin{tabular}{|c|c|c|c|c|c|c|}
\hline \multirow[t]{2}{*}{ Genotipo } & \multirow[t]{2}{*}{ Origen $^{A}$} & \multicolumn{3}{|c|}{$\begin{array}{c}\text { Campo } \\
\text { Promedio semestral }\end{array}$} & \multicolumn{2}{|c|}{$\begin{array}{c}\text { Evaluación } \\
\text { en invernadero }\end{array}$} \\
\hline & & 1998B & 1999A & Reacción $B$ & $\operatorname{Raza} 651^{B}$ & $\operatorname{Raza} 653^{B}$ \\
\hline $\begin{array}{l}\text { ICA Viboral } \\
\text { (testigo) }\end{array}$ & Rionegro & 7,0 & 8,0 & S & S & $S$ \\
\hline G-1 & Marinilla & 8,0 & 7,5 & S & S & $\mathrm{S}$ \\
\hline G-3 & C. Viboral & 6,5 & 5,5 & I & $\mathrm{S}$ & $S$ \\
\hline G-4 & C. Viboral & 8,0 & 7,0 & S & $\mathrm{S}$ & $\mathrm{R}$ \\
\hline G-5 & Guarne & 8,5 & 6,5 & S & $\mathrm{R}$ & I \\
\hline G-6 & Guarne & 8,5 & 5,5 & $\mathrm{~S}$ & $\mathrm{~S}$ & $\mathrm{R}$ \\
\hline G-7 & S. Vicente & 6,0 & 8,0 & $\mathrm{~S}$ & $\mathrm{~S}$ & $\mathrm{R}$ \\
\hline G-8 & S. Vicente & 8,5 & 5,0 & $\mathrm{~S}$ & $\mathrm{~S}$ & $\mathrm{~S}$ \\
\hline G-9 & Marinilla & 7,5 & 9,0 & $\mathrm{~S}$ & $\mathrm{~S}$ & $\mathrm{R}$ \\
\hline G-11 & C. Viboral & 8,0 & 9,0 & $\mathrm{~S}$ & $\mathrm{~S}$ & $\mathrm{~S}$ \\
\hline
\end{tabular}

A: Municipio productor en donde se colectó.

B: La reacción de las plantas a la inoculación con Colletotrichum lindemuthianum se basó en la escala CIAT (1: Sin síntomas aparentes de la enfermedad; 9: Síntomas severos o muerte; donde los grados 1 a 3 se designan como resistentes (R); la escala de valores entre 4 y 6 se designó como intermedia (I) y los valores entre 7 y 9 se designaron como susceptible (S)). 
que explica de algún modo la presencia de genotipos cultivados comercialmente sin resistencia a enfermedades transmitidas por la semilla, como la antracnosis.

Las evaluaciones realizadas en el invernadero, confirman la susceptibilidad de los genotipos G-1, G-3, G-8 y G-11 a las razas 651 y 653 de $C$. lindemuthianum., mientras que los genotipos G-4, G-6, G-7 y G-9 presentaron resistencia a la raza 653 y el genotipo G-5 sólo mostró resistencia a la raza 651 . Los genotipos G4, G5, G6, G7 y G9 podrían ser usados como potenciales fuentes de resistencia a la raza 653 o en investigaciones que estén interesadas en piramidar genes. Así mismo, al realizar las evaluaciones individuales de las 10 plantas inoculadas en condiciones de invernadero con el mismo aislamiento, se observó respuestas de completa susceptibilidad o resistencia (Cuadro 2). Una nueva evaluación de los genotipos 64, G-5, G-6, G-7, G-8, y G-9 mostró los mismos resultados; éstos podrían presentar mezcla con semillas muy similares al igual que los genotipos que presentan reacciones intermedias a las inoculaciones con las razas 651 y 653 , dado que fueron colectados directamente de las fincas. La venta de la semilla entre productores de la misma zona o zonas productoras diferentes (Quirós et al. 1997a, 1997b) y la variabilidad entre las semillas de cultivares comerciales tipo Cargamanto (Moreno y Elejalde, 1994) facilitan la mezcla de las semillas de las próximas siembras y la diseminación de las razas de antracnosis en las zonas productoras.
De otra parte, la reacción de susceptibilidad de los genotipos comerciales en condiciones de campo está enmascarando la resistencia que pueden tener estos mismos genotipos a por lo menos una raza de antracnosis prevalente en la zona, siendo necesario realizar las evaluaciones en invernadero y campo antes de ser descartados como posibles fuentes de resistencia. Además, es necesario continuar la colecta e identificación de la variabilidad del patógeno, realizar nuevas colectas de genotipos de frijol tipo Cargamanto e identificar los materiales que presentan resistencia a por lo menos una raza de antracnosis.

Este conocimiento sugiere el uso de los cultivares de agricultor, con tipo de semilla comercial y culturalmente con alta preferencia, en los programas de mejoramiento genético e introgresión de genes de resistencia de otras fuentes, aumentando la variabilidad genética de los frijoles Cargamanto. Igualmente podría ser útil colectar líneas establecidas como resistentes para purificarlas.

\section{Genotipos procedentes del banco de germoplasma de CORPOICA}

Las evaluaciones realizadas en condiciones de campo muestran que los genotipos LAS 73, LAS 106, LAS 331, LAS 384, ICA Llanogrande y Diacol Catío son resistentes a la antracnosis; respuestas similares fueron encontradas en la evaluación de variedades mejoradas (Román y Davis, 1986; Álvarez, 1972) y líneas

Cuadro 2. Evaluación de la severidad a la antracnosis de 13 genotipos procedentes del banco de germoplasma de CORPOICA. Antioquía, Colombia. 1999.

\begin{tabular}{|c|c|c|c|c|c|c|}
\hline \multirow[t]{2}{*}{ Genotipo } & \multirow[t]{2}{*}{ Tipo A } & \multicolumn{3}{|c|}{$\begin{array}{c}\text { Campo } \\
\text { Promedio semestral }\end{array}$} & \multicolumn{2}{|c|}{$\begin{array}{c}\text { Evaluación } \\
\text { en Invernadero }\end{array}$} \\
\hline & & 1998B & 1999A & Reacción $B$ & $\operatorname{Raza} 651^{B}$ & $\operatorname{Raza} 653^{B}$ \\
\hline ICA Viboral (testigo) & V & 7,0 & 8,0 & $S$ & $S$ & $\mathrm{~S}$ \\
\hline LAS 73 & $\mathrm{~V}$ & 3,0 & 1,6 & $\mathrm{R}$ & S & $\mathrm{S}$ \\
\hline LAS 106 & $\mathrm{~V}$ & 2,0 & 2,5 & $\mathrm{R}$ & $\mathrm{R}$ & $\mathrm{R}$ \\
\hline LAS 120 & $\mathrm{~V}$ & 6,5 & 5,0 & I & $\mathrm{S}$ & $\mathrm{S}$ \\
\hline LAS 129 & $\mathrm{~V}$ & 7,0 & 6,5 & $\mathrm{~S}$ & $\mathrm{R}$ & $\mathrm{S}$ \\
\hline LAS 331 & $\mathrm{~V}$ & 2,0 & 3,0 & $\mathrm{R}$ & $\mathrm{S}$ & $\mathrm{S}$ \\
\hline LAS 384 & $\mathrm{~V}$ & 3,0 & 2,0 & $\mathrm{R}$ & S & $\mathrm{R}$ \\
\hline LAS 435 & V & 4,5 & 2,5 & I & $\mathrm{S}$ & $\mathrm{S}$ \\
\hline LAS 614 & $\mathrm{~V}$ & 7,0 & 8,0 & S & $\mathrm{S}$ & $\mathrm{S}$ \\
\hline LAS 618 & $\mathrm{~V}$ & 7,5 & 7,5 & $\mathrm{~S}$ & $\mathrm{~S}$ & $\mathrm{~S}$ \\
\hline ICA Llanogrande & V & 3,5 & 3,0 & $\mathrm{R}$ & $\mathrm{S}$ & $\mathrm{S}$ \\
\hline Frijolica L.S.3.3 & $\mathrm{V}$ & 5,5 & 5,5 & I & $\mathrm{R}$ & $S$ \\
\hline Mocho La Ceja (testigo) & A & 6,5 & 4,0 & I & $\mathrm{R}$ & $\mathrm{S}$ \\
\hline Diacol Catío & A & 3,0 & 2,5 & $\mathrm{R}$ & $\mathrm{R}$ & $\mathrm{R}$ \\
\hline
\end{tabular}

A Tipo de crecimiento: $\mathrm{V}=$ voluble; $\mathrm{A}=$ arbustivo

B La reacción de las plantas a la inoculación con Colletotrichum lindemuthianum se basó en la escala CIAT (1: Sin síntomas aparentes de la enfermedad; 9: Síntomas severos o muerte; donde los grados 1 a 3 se designan como resistentes (R); la escala de valores entre a 4 y 6 se designó como intermedia (I) y los valores entre 7 y 9 se designaron como susceptibles(S). 
avanzadas del programa de mejoramiento de frijol de CORPOICA (Ríos, 1997). Los otros genotipos fueron tan susceptibles como el testigo ICA Viboral (Cuadro 3).

En cuanto a las evaluaciones en invernadero, los genotipos LAS 129, Frijolica L.S-3.3 y la variedad regional Mocho La Ceja mostraron resistencia a la raza 651 y el genotipo LAS 384 fue resistente la raza 653 (Cuadro 3). Los genotipos LAS 106 y Diacol Catío fueron resistentes en invernadero a las dos razas inoculadas y en condiciones de campo, por lo tanto podrían ser usados como fuentes de resistencia a la antracnosis. Igualmente genotipos que mostraron resistencia a una raza en condiciones de invernadero o en campo tienen potencial para ser utilizados en estudios de resistencia que pretendan piramidar genes. Los resultados indican que algunos genotipos pueden ser resistentes a la antracnosis en condiciones de campo aunque pueden ser susceptibles en condiciones de invernadero a las dos razas más virulentas encontradas en el Oriente Antioqueño; esto puede deberse a que ninguna de las dos razas se encontraban presentes en el sitio de siembra o que hubo diferentes respuestas de resistencia dependiendo de la edad de la planta. También hubo genotipos que presentaron resistencia a una de las dos razas bajo condiciones de invernadero aunque en el campo se observaron como susceptibles, lo que estaría mostrando la gran variabilidad de razas que pueden encontrarse en condiciones naturales. Estos resultados indican que las evaluaciones realizadas en invernadero no son totalmente confiables para buscar fuentes de resistencia a la antracnosis cuando se usan las razas 651 y 653, debido a que estas dos razas representan solamente una fracción de la variabilidad patogénica que se encuentra en Antioquía. Sin embargo, los ensayos realizados en invernadero con esta dos razas pueden ser usados como una manera rápida para descartar material susceptible. Los

Cuadro 3. Evaluación de la severidad a antracnosis de 26 genotipos del banco de germoplasma del CIAT. Antioquía, Colombia. 1999.

\begin{tabular}{|c|c|c|c|c|c|c|}
\hline \multirow[t]{2}{*}{ Genotipo } & \multirow[t]{2}{*}{ Tipo $^{A}$} & \multicolumn{3}{|c|}{$\begin{array}{c}\text { Campo } \\
\text { Promedio semestral }\end{array}$} & \multicolumn{2}{|c|}{$\begin{array}{c}\text { Evaluación } \\
\text { en Invernadero }\end{array}$} \\
\hline & & 1998B & 1999A & Reacción'B & Raza $651^{B}$ & $\operatorname{Raza} 653^{B}$ \\
\hline ICA Viboral (testigo) & V & 7,0 & 8,0 & $\mathrm{~S}$ & S & $\mathrm{S}$ \\
\hline CC21148 -4-1 & $\mathrm{V}$ & 7,5 & 7,0 & $\mathrm{~S}$ & S & $\mathrm{S}$ \\
\hline CC21148 -25-2 & $\mathrm{V}$ & 9,0 & 7,5 & $\mathrm{~S}$ & $\mathrm{~S}$ & $\mathrm{~S}$ \\
\hline CC21148 -51-1 & $\mathrm{V}$ & 7,5 & 7,0 & $\mathrm{~S}$ & $\mathrm{~S}$ & $\mathrm{~S}$ \\
\hline CC21148 -67-1 & $\mathrm{V}$ & 7,5 & 6 & $\mathrm{~S}$ & $S$ & $\mathrm{~S}$ \\
\hline CC21148 -74-1 & $\mathrm{V}$ & 7,5 & 6,5 & $\mathrm{~S}$ & S & $\mathrm{S}$ \\
\hline CC21148 -88-1 & $\mathrm{V}$ & 6,0 & 2,0 & I & $S$ & $\mathrm{~S}$ \\
\hline CC21148 -218-3 & V & 9,0 & 3,5 & $\mathrm{~S}$ & $S$ & $\mathrm{~S}$ \\
\hline CC21148 -R-5-1 & $\mathrm{V}$ & 8,5 & 8,0 & $\mathrm{~S}$ & $\mathrm{~S}$ & $\mathrm{~S}$ \\
\hline CC21148 -R-59-1 & $\mathrm{V}$ & 8,0 & 9,0 & $\mathrm{~S}$ & $\mathrm{~S}$ & $\mathrm{~S}$ \\
\hline CC21148 -R-67-3 & V & 8,0 & 9,0 & $\mathrm{~S}$ & $S$ & $\mathrm{~S}$ \\
\hline CC21148 -R-76-1 & $\mathrm{V}$ & 6,0 & 6,5 & I & S & $\mathrm{S}$ \\
\hline CC21148 -R-191-1 & $\mathrm{V}$ & 6,5 & 6,5 & I & $S$ & $\mathrm{~S}$ \\
\hline CC21148 -R-122-1 & V & 8,5 & 8,5 & $\mathrm{~S}$ & $S$ & $\mathrm{~S}$ \\
\hline AND 1080 & $\mathrm{~V}$ & 4,0 & 4,0 & $\mathrm{I}$ & $\mathrm{S}$ & $\mathrm{S}$ \\
\hline AND 1083 & $\mathrm{~V}$ & 5,0 & 3,5 & I & $\mathrm{S}$ & $\mathrm{S}$ \\
\hline AND 1084 & $\mathrm{~V}$ & 3,0 & 3,0 & $\mathrm{R}$ & $\mathrm{R}$ & $\mathrm{R}$ \\
\hline Mocho La Ceja (testigo) & A & 6,5 & 4,0 & I & $\mathrm{R}$ & $\mathrm{S}$ \\
\hline SUG 130 & A & 3,0 & 1,0 & $\mathrm{R}$ & $\mathrm{R}$ & $\mathrm{R}$ \\
\hline SUG 143 & $\mathrm{~A}$ & 5,0 & 2,5 & I & - & - \\
\hline SEQ 1035 & $\mathrm{~A}$ & 4,5 & 2,0 & I & $\mathrm{R}$ & $\mathrm{R}$ \\
\hline SEQ 1040 & $\mathrm{~A}$ & 3,0 & 2,5 & $\mathrm{R}$ & $\mathrm{S}$ & $\mathrm{R}$ \\
\hline FOT 28 & A & 4,0 & 2,0 & I & $\mathrm{S}$ & $\mathrm{S}$ \\
\hline FOT 29 & $\mathrm{~A}$ & 5,5 & 3,5 & I & $\mathrm{R}$ & $\mathrm{S}$ \\
\hline FOT 54 & A & 3,5 & 2,5 & I & $\mathrm{S}$ & $\mathrm{S}$ \\
\hline FOT 59 & $\mathrm{~A}$ & 5,0 & 1,0 & I & $\mathrm{R}$ & $\mathrm{S}$ \\
\hline PR 93201473 & A & 7,0 & 1,5 & $\mathrm{~S}$ & $\mathrm{R}$ & $\mathrm{R}$ \\
\hline PR 93201474 & A & 5,5 & 1,5 & I & $\mathrm{R}$ & $\mathrm{R}$ \\
\hline
\end{tabular}

A: Tipo de crecimiento: $\mathrm{V}=$ voluble; $\mathrm{A}=$ arbustivo

B: La reacción de las plantas a la inoculación con Colletotrichum lindemuthianum se basó en la escala CIAT (1: Sin síntomas aparentes de la enfermedad; 9: Síntomas severos o muerte; donde los grados uno a tres se designan como resistentes $(\mathrm{R})$; la escala de valores entre a 4 y 6 se designó como intermedia (I) y los valores entre 7 y 9 se designaron como susceptibles (S).

- : No hubo semilla disponible. 
genotipos resistentes pueden entonces ser sometidos a la amplia variabilidad genética del patógeno que existe en condiciones naturales o de campo. De esta manera se asegurará que las posibles fuentes de resistencia sean sometidas a la amplia variabilidad genética de $C$. lindemuthianum que existe en el campo y que la selección de genes de resistencia tengan un ámbito más amplio de actividad, lo que será útil para piramidar genes en los diferentes programas de mejoramiento genético. Debido a que los genotipos poseen uno o varios genes que le confieren resistencia a una de las dos razas y dada la amplia variabilidad genética que presenta $C$. lindemuthianum (Otoya et al. 1995), estos genotipos pueden ser susceptibles a otras razas del hongo presentes en el campo. Así mismo, se encontraron genotipos resistentes en condiciones de campo y de invernadero, los cuales pueden poseer genes de resistencia no solo para las dos razas evaluadas sino para otras que se encuentren presentes con mayor frecuencia en condiciones naturales.

\section{Genotipos procedentes del banco de germoplasma del CIAT}

Las evaluaciones realizadas en campo mostraron la irregular distribución del inóculo y el efecto del ambiente una vez que los genotipos CC 21148-88-1, SUG 143, SEQ 1035, FOT 28, FOT 54, FOT 59,
PR93201473 y PR 93201474 tuvieron reacciones de resistencia en un semestre y de susceptibilidad en otro (Cuadro 3). Esta doble reacción a la antracnosis en líneas homocigotas, puede ser debida a la existencia de varios factores que influyen en la reacción de estos genotipos en condiciones de campo. La infección del hongo ocurre bajo condiciones ambientales de alta humedad relativa (mayor a 92\%), con una temperatura promedio de $17^{\circ} \mathrm{C}$ y a la diseminación de las conidias en el campo que ocurre por lluvias moderadas, por el movimiento de insectos, animales o el hombre (Chaves 1989). La ausencia de uno o varios de estos factores inciden en la inoculación natural y al azar de las plantas, por lo que estos genotipos son susceptibles a la antracnosis. De otra parte, se observó resistencia en condiciones de campo en los genotipos AND 1084, SUG 130 y SEQ 1040.

En las evaluaciones de invernadero los genotipos AND 1084, SUG 130, SEQ 1035, PR 932011473 y PR 93201474 presentaron resistencia a las razas 651 y 653 (Cuadro 4). Los genotipos AND 1084 y SUG 130 fueron resistentes a las condiciones de campo e invernadero, por lo tanto son una fuente potencial de resistencia que pueden ser utilizadas para controlar la antracnosis. Sin embargo es importante que la resistencia de estos materiales sea evaluada en diferentes lugares donde la antracnosis se presenta como un problema severo, a fin

Cuadro 4. Fenotipo de virulencia y raza de aislamientos de Colletotrichum lindemuthianum provenientes de Rionegro -Antioquía, Colombia. 1999.

\begin{tabular}{|c|c|c|c|c|c|c|c|c|c|c|c|c|c|c|}
\hline \multirow[b]{2}{*}{ Aislamiento } & \multirow[b]{2}{*}{ Raza } & \multirow[b]{2}{*}{$\mathbf{A}$} & \multirow[b]{2}{*}{$\mathbf{B}$} & \multicolumn{7}{|c|}{ Variedades Diferenciales } & \multirow[b]{2}{*}{$\mathbf{J}$} & \multirow[b]{2}{*}{$\mathbf{K}$} & \multirow[b]{2}{*}{$\mathbf{L}$} & \multirow[b]{2}{*}{ Genotipo } \\
\hline & & & & $\mathbf{C}$ & D & $\mathbf{E}$ & $\mathbf{F}$ & $\mathbf{G}$ & $\mathbf{H}$ & $\mathbf{I}$ & & & & \\
\hline CL-272-COL & 1 & a & & & & & & & & & & & & G 5 \\
\hline CL-280-COL & 1 & $\mathrm{a}$ & & & & & & & & & & & & CC21148-R-5-1 \\
\hline CL-273-COL & 5 & a & & $\mathrm{c}$ & & & & & & & & & & G6 \\
\hline CL-283-COL & 5 & $\mathrm{a}$ & & $\mathrm{c}$ & & & & & & & & & & CC21148-R-59-1 \\
\hline CL-271-COL & 9 & $\mathrm{a}$ & & & $\mathrm{d}$ & & & & & & & & & G 1 \\
\hline CL-284-COL & 129 & a & & & & & & & $\mathrm{h}$ & & & & & ICA VIBORAL \\
\hline CL-278-COL & 131 & $\mathrm{a}$ & $\mathrm{b}$ & & & & & & $\mathrm{h}$ & & & & & G8 \\
\hline CL-281-COL & 131 & $\mathrm{a}$ & $\mathrm{b}$ & & & & & & $\mathrm{h}$ & & & & & CC21148-R-67-3 \\
\hline CL-288-COL & 131 & a & $\mathrm{b}$ & & & & & & $\mathrm{h}$ & & & & & LAS 614 \\
\hline CL-282-COL & 135 & $\mathrm{a}$ & $\mathrm{b}$ & $\mathrm{c}$ & & & & & $\mathrm{h}$ & & & & & CC21148-25-2 \\
\hline CL-286-COL & 139 & $\mathrm{a}$ & $\mathrm{b}$ & & $\mathrm{d}$ & & & & $\mathrm{h}$ & & & & & LAS 435 \\
\hline CL-274-COL & 513 & a & & & & & & & & & $\mathrm{j}$ & & & CC21248-218-3; LAS 120 \\
\hline CL-279-COL & 641 & $\mathrm{a}$ & & & & & & & $\mathrm{h}$ & & $\mathrm{j}$ & & & G 9 \\
\hline CL-277-COL & 643 & $\mathrm{a}$ & $\mathrm{b}$ & & & & & & $\mathrm{h}$ & & $\mathrm{j}$ & & & G 7 \\
\hline CL-275-COL & 645 & $\mathrm{a}$ & & $\mathrm{c}$ & & & & & $\mathrm{h}$ & & $\mathrm{j}$ & & & CC21148-4-1 \\
\hline \multicolumn{15}{|c|}{ Razas inoculadas } \\
\hline & 651 & $\mathrm{a}$ & $\mathrm{b}$ & & $\mathrm{d}$ & & & & $\mathrm{h}$ & & $\mathrm{j}$ & & & \\
\hline & 653 & a & & $\mathrm{c}$ & $\mathrm{d}$ & & & & $\mathrm{h}$ & & $\mathrm{j}$ & & & \\
\hline
\end{tabular}

Interacciones compatibles son designadas con una letra minúscula. Si la interacción es incompatible se deja el espacio en blanco.

La designación de la raza se obtiene sumando los valores de las variedades susceptibles. Raza $9(1+8)$; Raza $645(1+4+$ $128+512)$. 
de establecer su adaptabilidad a estas regiones. Es importante tener en cuenta que los genes de estos genotipos podrían ser introducidos a variedades comerciales, tanto solos como combinados.

El bajo número de genotipos resistentes (7,5\%) encontrados en este estudio, además de los reportados por Tamayo et al. (1995), sugiere que es necesario continuar la evaluación de fuentes de resistencia a antracnosis, especialmente de la amplia variabilidad que existe en el banco de germoplasma del CIAT reportada por Debouck (1999) y que está disponible para su uso por parte de los programas de investigación (Singh 1992).

\section{CONCLUSIONES}

Los resultados de este estudio muestran que:

Entre los 15 aislamientos colectados en el Centro de Investigación La Selva en Rionegro (Antioquía), 11 razas fueron identificadas, lo cual demuestra la amplia variabilidad genética de $C$. lindemuthianum en esta zona. Seis nuevas razas fueron caracterizadas por primera vez en Antioquía $(9,131,135,139,643$, y 645). Las razas $131,135,139,643$, y 645 fueron identificadas por primera vez en Colombia. Existe por lo tanto la necesidad de continuar con la caracterización de la variabilidad genética de $C$. lindemuthianum y la distribución geográfica del patógeno en esta región, dado que ésto permitirá la identificación de genes de resistencia y el desarrollo de estrategias de control de la enfermedad. Debido a la amplia variabilidad genética del patógeno en la zona, las posibles fuentes de resistencia deben ser buscadas en condiciones de campo y evaluadas en invernadero con respecto a las dos razas más virulentas de la región, de esta forma se podrá eliminar los genotipos susceptibles y establecer la durabilidad de los resistentes. Los materiales que resulten resistentes podrán ser examinados en condiciones de campo para verificar la utilidad de la resistencia identificada.

Muy poca o ninguna resistencia a la antracnosis existe en las variedades comerciales de frijol cultivadas en Antioquía. La mayoría de los genotipos tienen reacciones combinadas en condiciones de invernadero; mientras que algunas plantas se muestran muy resistentes, otras presentan alta susceptibilidad. Probablemente debido a las mezclas de semillas de la finca productora y de otras fincas de otras zonas con el mismo tipo de semilla realizadas por los productores. Se hace necesario entonces purificar estos genotipos y evaluarlos nuevamente en invernadero y en campo antes de ser identificados como susceptibles o resistentes.
La resistencia encontrada a por lo menos una de las dos razas inoculadas en los genotipos comerciales, hace necesario continuar con la colecta y evaluación de genotipos regionales que pueden ser fuente de resistencia a razas de antracnosis prevalentes en Antioquía, así como de fuente de conocimiento de la variabilidad del patógeno. Además, es pertinente la evaluación de nuevos viveros de los bancos de germoplama del CIAT y CORPOICA.

Dos genotipos procedentes del banco de germoplasma de CORPOICA (LAS 106 y Diacol Catío) mostraron resistencia a las dos razas más virulentas $(651$ y 653) reportadas en Antioquía en condiciones de invernadero y al amplio rango de patotipos en condiciones de campo. Entre los genotipos procedentes del CIAT, AND 1084 y SUG 130 presentaron resistencia a $C$. lindemuthianum en condiciones de invernadero y de campo. Los cuatro genotipos conforman una fuente potencial de resistencia a la antracnosis que pueden ser usados directamente o introduciendo genes de resistencia, solos o en diferentes combinaciones, en variedades comerciales. Así mismo, es importante evaluar estos materiales en otras localidades de Rionegro, Antioquía y de Colombia, donde la antracnosis tiene alta incidencia a fin de establecer su rango de actividad.

Debido al alto grado de variabilidad que presenta el patógeno, en el desarrollo de las nuevas variedades de frijol Cargamanto se sugiere la piramidación de genes como un estrategia para obtener una resistencia duradera, no obstante es necesario tener en cuenta que los procedimientos convencionales implican inoculaciones múltiples para la selección, mientras que el uso de marcadores moleculares puede aumentar la eficiencia de la selección.

\section{AGRADECIMIENTOS}

Al Proyecto de Frijol para la Zona Andina (PROFRIZA) por la financiación y al proyecto de frijol del Centro Internacional de Agricultura Tropical (CIAT) por el apoyo técnico y científico.

\section{LITERATURA CITADA}

ALVAREZ, J. 1972. Leguminosas de grano. In: Informe anual de actividades. Instituto Colombiano Agropecuario (ICA).Rionegro, Antioquía, Colombia. pp 29-31.

ARIAS, J.; MONSALVE, F. 2000. Producción y manejo de semilla de frijol para pequeños productores del municipio de Urrao. Centro de Investigación La Selva. 
Corporación Colombiana de Investigación Agropecuaria (CORPOICA), Regional 4. Rionegro , Antioquía, Colombia Informe técnico. $30 \mathrm{p}$.

CHAVES, G. 1980. La antracnosis. In: H. Schwartz y G. Gálvez eds. Problemas de producción del frijol. Centro Internacional de Agricultura Tropical (CIAT). Cali, Colombia. pp. 37-53.

CHAVES, G. 1989. Anthracnose. In:H. Schwartz and G. Gálvez eds. Bean production problems. Centro Internacional de Agricultura Tropical (CIAT). Cali, Colombia. pp. 39-54.

DEBOUCK, D. 1999. Diversity in Phaseolus species in relation to the common bean. In Singh, S.P. ed. Common bean improvement in the twenty-first century. Kluwer, Dordrecht, Netherlands. pp. 25-52.

MORENO, F.; ELEJALDE, G. 1994. Caracterización fenotípica de frijoles tipo Cargamanto Phaseolus vulgaris L. Tesis de pregrado. Facultad de Ciencias Agropecuarias. Departamento de Agronomía. Universidad Nacional de Colombia, sede Medellín, Colombia.135p.

OTOYA, M.; RESTREPO, S.; PASTOR, M. 1995. Amplificación al azar del ADN polimórfico para evaluar la diversidad genética de Colletotrichum lindemuthianum en Colombia. Fitopatología Colombiana. 19(1):7-14.

PASTOR-CORRALES, M. A.; TU, J.C. 1989. Anthracnose. In: Schwartz, H.F. and M.A. Pastor eds. $2^{\text {nd }}$. Bean Production Problems in the Tropics. Centro Internacional de Agricultura Tropical (CIAT), Cali, Colombia. pp.77-104

PASTOR, M.A 1992. Variación patogénica de Colletotrichum lindemuthianum, el agente causal de la antracnosis del frijol y una propuesta para su estandarización. In: M.A. Pastor ed. La antracnosis del frijol común, Phaseolus vulgaris en América Latina. Centro Internacional de Agricultura Tropical (CIAT), Cali, Colombia. Working Document, Bean Program No. 113. pp 212-239.

PASTOR, M.A., OTOYA, M.M.; MAYA, M.M. 1993. Diversidad de la virulencia de Colletotrichum lindemuthianum en Mesoamérica y la Región Andina. Fitopatología Colombiana 17:31-38.

QUIROS, J.; AREVALO, M.; MUNERA, G.; DIAZ, C.; RIOS, M. 1996. Factores determinantes de la adopción de tecnología de frijol en Urrao, Antioquía. Centro de Investigación La Selva. Corporación Colombiana de Investigación Agropecuaria (CORPOICA), Regional 4. Rionegro, Antioquía, Colombia. Boletín de investigación No. 2.83 p.

QUIROS, J.; AREVALO, M.; DIAZ, C.; RIOS, M. 1997a. Factores relacionados con la adopción de tecnología en el cultivo de frijol en Uramita, Antioquía. Centro de Investigación La Selva. Corporación Colombiana de Investigación Agropecuaria (CORPOICA), Regional 4.
Rionegro, Antioquía, Colombia. Boletín de investigación No. 5.79 p.

QUIROS, J. ; AREVALO, M.; DIAZ, C.; RIOS, M. 1997b. Limitantes de la adopción de tecnología en el cultivo de frijol en Dabeiba, Antioquía. Centro de Investigación La Selva. Corporación Colombiana de Investigación Agropecuaria (CORPOICA), Regional 4. Rionegro, Antioquía, Colombia. Boletín de investigación No. 7.86 p.

RESTREPO, S. 1994. DNA polymorphism and virulence variation of Colletotrichum lindemuthianum in Colombia. M.Sc Thesis, Institut National Agronomique ParisGrignon, Universite Paris. VI. 42 p.

RÍOS, J. M. 1997. Líneas mejoradas de frijol voluble y arbustivas para el clima frío; obtenidas en el Centro de Investigación La Selva, programa de leguminosas ICA (Convenio ICA-CIAT). Rionegro, Antioquía, Colombia. Informe de trabajo. $26 \mathrm{p}$.

ROMÁN, A. 1992. El cultivo de frijol en el Oriente Antioqueño. In: M.A. Pastor ed. La antracnosis del frijol común (Phaseolus vulgaris) en América Latina. Centro Internacional de Agricultura Tropical (CIAT), Cali, Colombia. Documento de Trabajo No. 113. pp 170-72.

ROMÁN, A.; DAVIS, J. 1986. ICA Llanogrande nueva variedad de frijol resistente a la antracnosis. Instituto Colombiano Agropecuario (ICA). Rionegro, Antioquía, Colombia. Plegable divulgativo No. 166.

SCHOONNHOVEN, A.; PASTOR, M. 1987. Sistema estándar para la evaluación de germoplasma de frijol. Centro Internacional de Agricultura Tropical (CIAT). Cali, Colombia. 56p.

SINGH, S.P. 1989. Patterns of variation in cultivated common bean (Phaseolus vulgaris, Fabaceae). Econ. Bot. 43:39-57.

SINGH, S.P. 1992. Common bean improvement in the tropics. Plant Breed. Rev. 10:199-269.

TAMAYO, P. 1995. Manejo y control de las enfermedades del frijol voluble (Phaseolus vulgaris L.). Centro de Investigación La Selva, Corporación Colombiana de Investigación Agropecuaria (CORPOICA). Regional 4. Rionegro, Antioquía, Colombia. Boletín técnico. 39p.

TAMAYO, P., OTOYA, M.; PASTOR, M. 1995. Diversidad de razas de Colletotrichum lindemuthianum, el patógeno de la antracnosis de frijol, en Rionegro, Antioquía. Fitopatología Colombiana. 19(1):1-6.

VOYSEST, O. 1999. Un programa de leguminosas de grano para la Región Andina: planes para el futuro. Centro Internacional de Agricultura Tropical (CIAT). Cali, Colombia. $24 \mathrm{p}$. 